\title{
Fuzzy Logic Technique to Select Redundant Nodes and form Fault Free Node in Wireless Sensor Networks
}

\author{
Shradha V. Hulamani, Kiran M. R, Hemantaraj M. Kelagadi, Dr. Priyatamkumar \\ School of Electronics and Communication \\ KLE Technological University Hubballi-580031(India)
}

\begin{abstract}
In Wireless sensor networks, energy dissipation occurs due to high degree of compactness in nodes.Sensor nodes contain less energy and memory, therefore it is necessary to maintain energy level of nodes.It can be achieved by using Fuzzy logic which groups the data based on similarity and put nodes to sleep mode to balance the energy.After Transmission of data in various rounds their may be node failure and link failure which need to be replaced early for maintaining the performance in network.This can be achieved by making use of Markov model and Fuzzy logic to detect the nodes and replace them quickly.The simulation results show the overall improvement in performance of nodes.
\end{abstract}

Index Terms-Fuzzy logic, TDMA, Markov model.

\section{INTRODUCTION}

Wireless Sensor Network are low cost and low power small device extensively used for sensing the environment .It can be defined as network of sensor devices that gather information from the environment and forward it to Base Station. Network is made up of number of sensing nodes which monitor environmental factors like temperature, sound pressure and pass the information by single hop or multiple hop. It is used in variety of applications like military surveillance and health monitoring etc. A large amount of energy consumption happens in data collection and transmission; hence selection of routing protocol is very important to optimize the energy consumption. In this paper, Hierarchical clustering topology gather information more efficiently. In such scenario, Network is split into few groups called Clusters .Each cluster has a Cluster-Head $(\mathrm{CH})$ which collects the information and transfer to Base-Station. By periodically changing the $\mathrm{CH}$ the energy consumption can be balanced. To reduce the energy consumption radius is computed where nodes are divided into cluster .Based on similarity of data the nodes, few nodes are put to sleep mode using fuzzy logic. Data transmission in Wireless sensor network is done using TDMA scheduling. The Objective of TDMA is to maximize the time-slot when redundant nodes are in sleep mode by this the nodes which have high information can use the time-slot of sleepy node. In Wireless sensor network there are cases when there is node fault and link fault due to energy depletion. In addition the fault nodes cause network coverage and overall network paralyze. Therefore in order to have good quality service, it is must to identify the nodes and link fault and design for recovery of those faults. Fault detection helps in isolating fault sensors and can respond to unpredicted failure. In this paper, Markov model is used to detect the faults and by defining the probability condition the recovery of fault is described. Thus the main contribution of this paper is:

- By gathering the data from the environment and by using the fuzzy logic we select certain redundant nodes based on data similarity and Nodes with high energy level are selected for transmission and other nodes are put into sleep mode to save the energy.

- Dynamic TDMA Scheduling provide time-slot for every node at every level for data transmission. TDMA used here is dynamic . The advantage of using dynamic TDMA is the time-slots are flexible therefore no information is lost if the nodes have large amount of data.

- Fault-tolerant is used to detect number of fault nodes. Strong fault-tolerant node and Weak faulttolerant node. Strong fault nodes manage to transfer the information whereas weak fault cannot and they need to be replaced by other sensor depending on the energy levels of the sensor. Using Markov model we check the reliability of every node and link and then develop fault free network.

\section{RELATED WORK}

Clustering is most efficient technique in Wireless Sensor Networks. In[1] it has been shown that to increase the stability and lifetime of the system design of energy efficient routing protocol is necessary. Selection of Cluster Head is done by monitoring the energy levels, Heterogeneous clustering scheme based on weight election probability .In[2]design and analysis of LEACH protocol for sensor network is done for data aggregation using concept of energy-efficient clustering protocol and media access together to achieve good lifetime, latency and quality of service .In[3]discussion of two major issues is done .First parameters optimization for WSN and consistency checking is done, Second to increase the lifetime framework of cross layer is incorporated. In[4] scheduling of sleep/wake for low-duty cycle sensor is discussed. In order to decrease the energy consumption and to provide high message delivery performance, formula is optimized to cut down energy consumption with limitation on the message delivery performance.In[5]improvement of unbalanced energy consumption among cluster head is seen.To solve the 
problem energy-efficient unequal clustering algorithm is proposed based on the distance from the Base Station.In[6]Sleep Scheduling and tree-based clustering for energy efficient is proposed. This algorithm saves energy by putting nodes in sleep mode. Minimum spanning tree further reduction of energy consumption is observed. In[7]Sleep Scheduling(SS)tree is proposed which help to reduce the energy consumption by monitoring the sensor capabilities, this paper aims to reduce various issues and provide solution to increase coverage and the operational life time of mesh based sensor network engaged in various surveillance application. In[8]optimized discharge curve based coverage protocol(ODCP) is proposed which help to determines the redundant nodes by using the information of neighbor nodes like battery discharge, overlap coverage information etc. In[9]Redundancy aware Topology Control Protocol(RTCP) is proposed which exploits the redundant nodes in the same region by dividing into groups which help to maintain the backbone connected by keeping few nodes working and few sleep. It helps to identify equivalent nodes communication based on information, it allows to keep one sensor node active from every group. In[10]discussion on how to ensure fault tolerance in WSN is seen, In this paper how to detect and recover the fault detected node is seen called as proactive node. Analysis show that analysis of up to fault nodes recovers depends on the threshold node redundancy. $\operatorname{In}[11]$ estimation of redundancy nodes is based on comprehensive calculation as dynamic matter, nodes with low redundancy weight force look at high redundancy weights and relocate .In[12]Markov model is developed to characterize Mean time to failure(MTTF) which helps to develop design for various applications .MTTF model could help WSN designers to better meet application necessity by determining MTTF in pre-deployment phase.In[13]To check failures, schemes have been proposed that perform task before a failure happens. Recovery approach have been proposed for single and multi nodes and the results are compared. In[14]routing algorithm in WSN for fuzzy logic is presented,In this paper capability to send data based on different energy levels, distance and load is determined. FL helps to determine and select packets for transmission. In[15] to reduce energy consumption novel based distribution approach has been demonstrated which for fuzzy numbers and weight average operators to perform aggregation which is energy efficient .In proposed algorithm it is shown that number of received and sent data can be reduced without effect the quality of services.In[16]BA model describes the distribution of the power law in networks which are complex in terms of dynamic and growing and develop scale free network . The discovery of scale-free properties paves a new way to enhance the invulnerability of network topology. In scale-free WSNs, the small proportion of sensor nodes possesses most connections of the network, and most of the sensor nodes are low-degree, hence it has high resistance.In[17]Model 1 is based upon scale-free and small complex theory and by taking into account characterstics of WSN ,a topology model with small and scall-free concept for heterogenous network is presented.In[18]Model 2 order-free topology for fault-tolerance and intrusion tolerance has been shown.The mathematical expression is derived .

\section{PROPOSED WORK}

Energy efficient clustering is important technique. Clusters consist of sensor nodes which are grouped in the cluster based on the coverage area. Data-transmission inside the cluster is known as intra-cluster and transmission between the clusters is known as inter-cluster. The cluster members sense the environment value and send to the Cluster Head $(\mathrm{CH})$ by multi-hoping i.e from Secondary parent node to Primary parent node. Figure 1. shown below depicts the network which is divided into several clusters for data transmission.

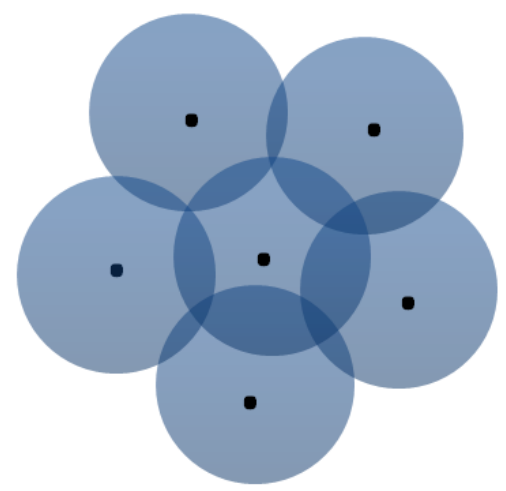

. indicate the Cluster Head

Figure 1: Cluster's in Wireless Sensor Network.

\section{A. Tree Topology}

We consider a tree topology with Parent -Child relation. The basic idea of clustering method is categorize the cluster based on the coverage area .Further the clusters are split into sectors based on the similarity of data as shown in Figure2. For example, single cluster is used to demonstrate the working. For a given tree, the policy that maximize the network lifetime is such that certain group of nodes become energy expended, while the other nodes have still sufficient energy unused. By proper selection of the tree so that each has a different group of expended nodes we obtain a scheduling algorithm which performs very well .In tree topology, the Child node $(\mathrm{CN})$ sense the environment data and sends to the Secondary parent node(SPN),SPN further aggregate the data and depending on the energy levels of primary parent node(PPN) it sends the data to the node which has high level of energy. The PPN sends the collected data to the $\mathrm{CH}$ in our example $\mathrm{CH} 1, \mathrm{CH} 1$ collects the data from various primary parent nodes and send to the sink, sink sends to the BS which is placed far away from the nodes sensing environment. TDMA scheduling is used at various levels of nodes to send the information.

The advantages of using Tree Topology are as follows:

1. The Parent nodes always keep tracking the status of the child node, and the connection status is aggregated. Therefore the connection status can be easily understood.

2. Other hierarchical networks are not affected if one of them is damaged.

3. Easier maintenance and easy fault detection. 
Fuzzy logic is made use in Tree Topology for splitting of nodes based on similarity and Radius is computed to divide the Network into numerous clusters in order to maintain balanced energy levels in the network.

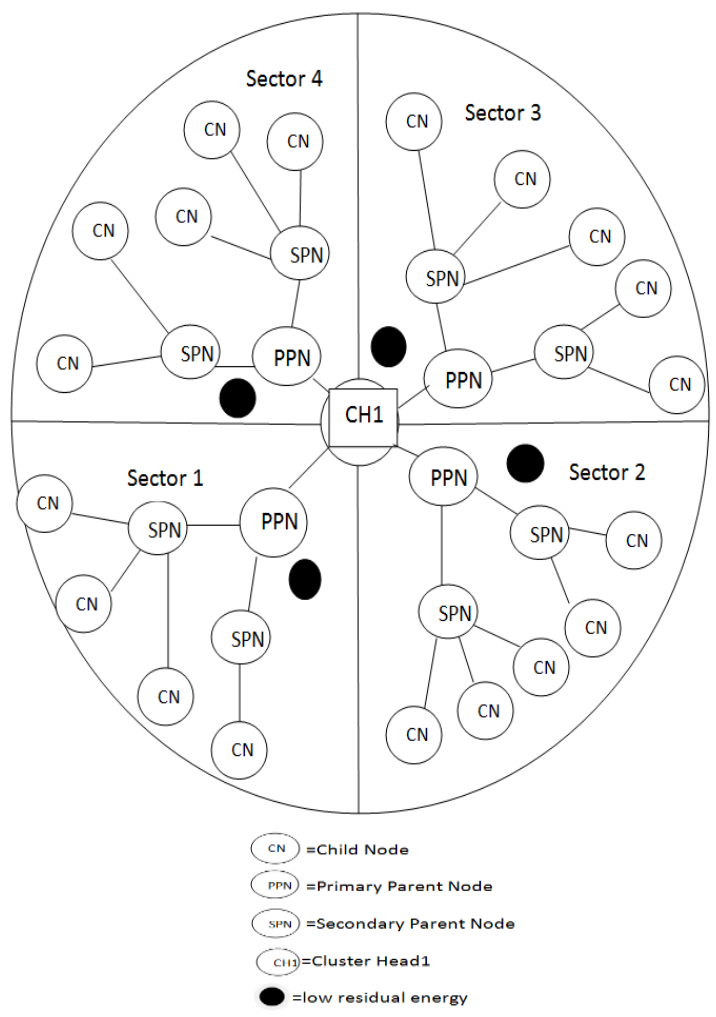

Figure 2: Single Cluster divided into Sectors

\section{B. Fuzzy logic}

Fuzzy logic is used for decision making which helps to reduce energy consumption. Data from the multiple resources are combined and grouped in order to get the conclusion by data fusion. This approach is more systematic and capable mean of achieving more specific data. The main motivation of data fusion is to get greater quality of service for reliability and for specific decisions. Fused data focuses on quality of service and energy consumption as it helps to eliminate redundant nodes in network. Fuzzy logic performs fuzzification, inference and defuzzification as shown in Figure 3. The fuzzification is process which transforms the crisp values to fuzzy linguistic variables. Crisp input data are put down together and converted to a fuzzy set of member functions, terms and linguistic variables . Afterwards, Inference computes the output corresponding to the rules. Eventually, the output is mapped to output using the membership functions in defuzzification [].

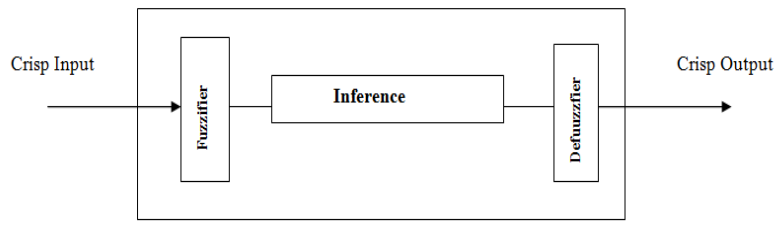

Figure 3. Fuzzy logic Block Diagram

For every crisp values taken it is converted into Member function which define certainty ,for example for temperature detection the linguistic value for -3 degree can be $30 \%$ frozen and $60 \%$ cold. fuzzy decision is made using certain rules which consists of rules having all possible combinations for linguistic values that constitute the surroundings. Defuzzification using the Maximum method the output value can be selected by considering the highest value of the Member function. Therefore by performing there different steps we select the redundant nodes which reduce the energy consumption[19].

\section{System Model}

\section{(i)Network model:}

Let us consider $N$ number of clusters consisting of nodes which are being distributed irregularly in the field area. The nodes overlook the area continuously and send the information to the BS periodically. Here, $s_{i}$ symbolizes the $i$ th sensor node, and set of sensor node is given by $\mathrm{S}=\left\{\mathrm{s}_{1}, \mathrm{~s}_{2}, \ldots . \mathrm{s}_{\mathrm{N}}\right\},|\mathrm{S}|=N$.

To decide the specifications(parameters) for our model, following speculations are made:

1. The position unaware sensors are split uniformly in network.

2. The BS is distant from the sensing field, located outside the sensing region.

3. The sensor node can calculate the rough distance from the sender by detecting the signal strength.

The energy utilization of the nodes in each round can be calculated.

The Energy First Order Radio model is drawn on to measure the energy consumption [20].The energy spent for sending of $k$-bit packet from the source to the next destination at a distance (d) can be defined as:

$$
\mathrm{E}_{\mathrm{TX}}=\left\{\begin{array}{l}
k \mathrm{E}_{\text {elec }}+k \varepsilon_{f s} \mathrm{~d}^{2}, \mathrm{~d}<\mathrm{d}_{0} \\
k \mathrm{E}_{\text {elec }}+k \varepsilon_{m p} \mathrm{~d}^{4}, \mathrm{~d} \geq \mathrm{d}_{0}
\end{array}\right.
$$

Eelec is dissipated energy, $\mathrm{d}$ is transmission distance. $\varepsilon_{f s}$ is free space factor and $\varepsilon_{m p}$ is multiple factor. $\mathrm{d}_{0}$ is distance threshold.

The distance threshold can be given as 


$$
\mathrm{d}_{0}=\sqrt{\varepsilon m p / \varepsilon f s}
$$

The depleted energy for receiving k-bit of the data is:

$$
\mathrm{E}_{\mathrm{RX}}(k, \mathrm{~d})=k \mathrm{E}_{\text {elec }}
$$

The depleted energy for data aggregation is given as:

$$
\mathrm{E}_{\text {aggr }}(k, \mathrm{~d})=\mathrm{E}_{d a}
$$

Where $\mathrm{E}_{d a}$ is data fusion factor.

\section{(ii)Computing Radius:}

The distance should be limited within the threshold $\mathrm{d}_{0}$ for inter-cluster communication to reduce the energy consumption. The $\mathrm{CH}$ can send the data directly if transmission is single hop,If the BS is distant from the $\mathrm{CH}$ it needs to obtain several multi-path and this will increase the energy consumption of the $\mathrm{CH}$ causing quick death of the node and the lifetime of the entire node will reduce .It is must to calculate and set the radius of nodes and form uniformly distributed radius to stabilize the energy consumption.

The energy consumption of nodes at different level is formularized below .

The energy utilization by the child nodes in the given range to transmit information to the Parent node is given by :

$$
\begin{array}{r}
\mathrm{E}_{\mathrm{T}}=\alpha \int_{d_{p n}}^{0} 2 \pi x \times \rho \times\left(k \mathrm{E}_{\text {elec }}+k \varepsilon_{f s} x^{2}\right) \mathrm{d} x \\
=\alpha k \pi \rho \times\left(\mathrm{E}_{\text {elec }} d_{P N}^{2}+\frac{1}{2} \varepsilon_{f S} d_{B S}^{4}\right)
\end{array}
$$

Here $2 \pi x \times \rho \times d x$ is amount of nodes in circular area, $\rho=\mathrm{N} / \mathrm{V}^{2}$ is area density, $\alpha=\frac{1-M}{(n-1)}$ percentage of active nodes.

For monitoring the data received from the nodes the energy consumption by each $\mathrm{PN}$ and $\mathrm{CH}$ is given by:

$$
\mathrm{E}_{\mathrm{M}}=\mathrm{k} \times\left(\pi r_{i}^{2} \rho-1\right) \times \mathrm{E}_{\mathrm{elec}}
$$

Where $r_{i}$ is circular area radius

The energy consumption by each $\mathrm{PN}$ and $\mathrm{CH}$ for data aggregation is given by:

$$
\mathrm{E}_{\mathrm{ag}}=\mathrm{k} \times \pi r_{i}^{2} \rho \times \mathrm{E}_{\mathrm{DA}}
$$

The energy consumption to forward the data to base station is given by:

$$
\mathrm{E}_{\mathrm{BS}}=k \mathrm{E}_{\mathrm{elec}} \mathrm{X} \mathrm{k} \varepsilon_{m p} R_{i}^{4}
$$

$\mathrm{Ri}$ is the radius distance

Therefore total energy utilization in cluster is given by:

$$
E_{\text {Cluster }}=E_{T}+E_{M}+E_{a g}+E_{B S}
$$

The average energy consumed by node is given by:

$$
\mathrm{E}_{\mathrm{avg}}=\mathrm{E}_{\mathrm{cluster}} / \pi r_{i}^{2} \rho
$$

Therefore the radius is given by:

$$
\mathrm{d}_{\mathrm{CH}}=\sqrt[4]{\frac{2 \varepsilon_{m p} p}{\alpha \pi \rho \varepsilon_{f S}}} \mathrm{~d}_{\mathrm{BS}}
$$

When a candidate $\mathrm{CH}$ broadcasts the message, the nodes within that range can receive the message from the respective $\mathrm{CH}$. The spatial distribution can be restricted within the limited radius during $\mathrm{CH}$ selection. Calculation of radius is important as it will directly relate to network lifetime. If number of clusters is few and radius is large it will result in more energy utilization due to high signal power which is used for transmission at longer distance. Thus computing of radius is important to reduce overhead and maintain balanced energy in network.

\section{(iii)Selection of Cluster Head:}

Choosing of $\mathrm{CH}$ rely on the radius and probability. Initially, all nodes are treated as ordinary node, based on the remaining energy and distance (with respect to the Base station) $\mathrm{CH}$ is selected. Nodes which are close by to the BS have higher probability of getting selected as $\mathrm{CH}$ compared to nodes which are at far distance[21-22].

Every node has chance of becoming Cluster Head based on their energy level at various rounds. The probability function is given by

$$
\mathrm{CH} \text { 's(i) }=\gamma \times \frac{d_{\max }-d_{\text {toBs }}(i)}{d_{\max }-d_{\min }}+(1-\gamma) \times \frac{\mathrm{E}_{\mathrm{re}}(\mathrm{i})}{\mathrm{E}_{\mathrm{ini}}}
$$

Where $d_{\max }$ is maximum distance, $\gamma$ is the constant parameter, $\mathrm{E}_{\mathrm{re}}$ is residual energy, $\mathrm{E}_{\mathrm{ini}}$ is initial energy.

The selected $\mathrm{CH}$ should have information of the neighbor nodes which are in defined communication range. The selected node modify their information once they are selected.

The $\mathrm{CH}$ node collects information like ID, energy levels of the adjacent nodes after which it broadcast the message to nodes. The nodes which are near by depending on the distance and strength of signal join the respective $\mathrm{CH}$. This reduces the energy consumption by the nodes and energy level is balanced in the entire network. Similarly the PPN and SPN are selected in the same manner.

After selection is done, sensor nodes send the information and data aggregation is done at different levels .

\section{(iv)Selection of Redundant nodes:}

Few sensor nodes are put to sleep mode, to reduce the energy consumption. The member nodes are often located in the adjacent region. Redundant node selection can be determined by using the Fuzzy logic by using optimization strategy[2]. Using fuzzy logic we can group the data with high similarity into the same classification. 
A finite number of sensor nodes will be put to sleep mode from all the section during the scheduling process. The data received by $\mathrm{CH}$ from sensor nodes is gathered to an extent, then construction of fuzzy logic is done to form clusters. The data combining accuracy is high and some nodes will be put to sleep mode from all the category. Finally, scheduling mechanism is applied to those redundant node to reduce traffic dispute and communication cost.

The nodes $\mathrm{s}_{\mathrm{i}}$ and $\mathrm{s}_{\mathrm{j}}$ are defined as Distance called Confidence, which is indicated as Dis $(i, j)$. The measurement value of the pair of sensor node is close, if the value is small .For different confidence levels results obtained are different based on the classification and then clustering diagram can be formed.

Assuming $S=\left\{s_{1}, s_{2} \ldots . . . s_{n}\right\}$ which denotes the sensor nodes in cluster and $\mathrm{n}$ is the defined as number ocluster.

The TDMA scheduling which determine scheduling time for nodes can be split into $\mathrm{m}$ intervals and $\mathrm{a}_{i j}$ describes the data collected from the nodes $\mathrm{s}_{j}$ at time $j . \mathrm{A}=\left(\mathrm{a}_{i j}\right)_{n x m}$ defines the original data.

After standardization, A is transformed to fuzzy logic. Primarily, the shift and standard deviation transformation is carried out and the element in the normalized data can be given as

$$
a_{i j}^{\prime}=\frac{a_{i j}-\overline{\mathrm{a}}_{j}}{b j}, \quad(i=1,2, \ldots . n, j=1,2, \ldots \ldots m)
$$

Where $\overline{\mathrm{a}} \mathrm{j}=\frac{1}{n} \sum_{i=1}^{n} a_{i j}, b_{j}=\sqrt{\frac{1}{n} \sum_{i=1}^{n}(a i j-\overline{\mathrm{a}} j)^{2}} \quad,(\mathrm{j}=1,2 \ldots \mathrm{m})$

For the unified dimension it is defined as

$$
a_{i j}^{\prime \prime}=\frac{a_{i j}^{\prime}-\min _{1 \leq i \leq n}\left(a_{i j}^{\prime}\right)}{\max _{1 \leq i \leq n}\left(a_{i j}^{\prime}\right)-\min _{1 \leq i \leq n}\left(a_{i j}^{\prime}\right)} \quad(\mathrm{j}=1,2 \ldots \mathrm{m})
$$

Thus the fuzzy logic $\mathrm{R}=\left(a_{i j}^{\prime \prime}\right)_{\mathrm{nxm}}$ can be achieved.

There should be minimum information loss according to the principle of redundant node selection.

The objective function for selecting redundant nodes can be given as:

$$
s_{*}^{v}=\arg \min \sum_{i=1}^{v} \operatorname{Dst}\left(s_{i}^{(v)}, s_{j}^{(v)}\right)
$$

where $s_{*}^{v}$ denote the redundant nodes in $v$ and $s_{i}^{(v)}$ denotes ith sensor node in $v$ category.

\section{(v) Scheduling Algorithm}

Energy Efficient Sleep Scheduling Mechanism(EESM) is dispersed mechanism for unequal clusters, it makes decisions for calculating radius and selecting the $\mathrm{CH}$. In order to calculate the radius for selection of cluster heads, EESM considers both residual energy and distance to the BS parameters. In addition, it takes help of fuzzy logic method to determine the radius based on a probabilistic model, which is employed for competition between candidate clusterheads.

The $(\mathrm{CH})$ broadcasts their information to the adjacent in the competition range(radius) along with the transmission power.
The information contains the $\mathrm{CH}-\mathrm{ID}$ and the residual energy after receiving the message the other cluster heads copy the candidate $\mathrm{CH}$-table. While $\mathrm{CH}$ receives all the information from the member nodes it will store the information and apply fuzz logic to select the redundant nodes.

Dynamic TDMA is used for scheduling, TDMA is divided into several slots for transmission of the data which is discussed in the next section.

\section{Algorithm to determine the sleep nodes}

For each $\mathrm{CH}$

1. Put up raw logic A

2. After the data is standardized, it is transformed into a fuzzy logic R.

3. The nodes are divided into $\mathrm{k}$ categories.

4. for each category $\mathrm{v}$

5. for each node $\mathrm{j}, \mathrm{k} € \mathrm{~s}^{(\mathrm{v})}$

6. Calculate $\operatorname{Del}\left(s_{i}^{(v)} s_{j}^{(v)}\right)$ between the data from node $s_{i}^{(v)}, s_{j}^{(v)}$;

7. $s_{*}^{(v)}=\arg \min \left\{\sum_{i=1}^{v} \operatorname{Del}\left(s_{i}^{(v)} s_{j}^{(v)}\right)\right.$

8. end for

9. end for

10. Obtain the redundant nodes set

\section{TDMA Scheduling}

Flow model:

The transmission are organized into data flow.Each data flow consists parent node(sink node) from each cluster which collects data from the child nodes using fuzzy logic ,the child node i.e the source node periodically sense the data from the surrounding and transmit it to the secondary parent node, the primary parent node which has high energy level receives the data and send to the $\mathrm{CH} 1$.

Figure 4 below shows the TDMA schedule. Every sensor node of tree topology are assigned with time slots and each node can deliver $k$-packets. In TDMA scheduling the time-slot is fixed for fixed number of packets. Therefore, dynamic TDMA scheduling is used for 2 main reasons.

a. If there are few packets in the buffer to that of the time-slot ,the sensor node will remain active till the end of time slot, this will lead to energy drain.

b. If there are packets with greater size to that of fixed time-slot, the data may be lost. 


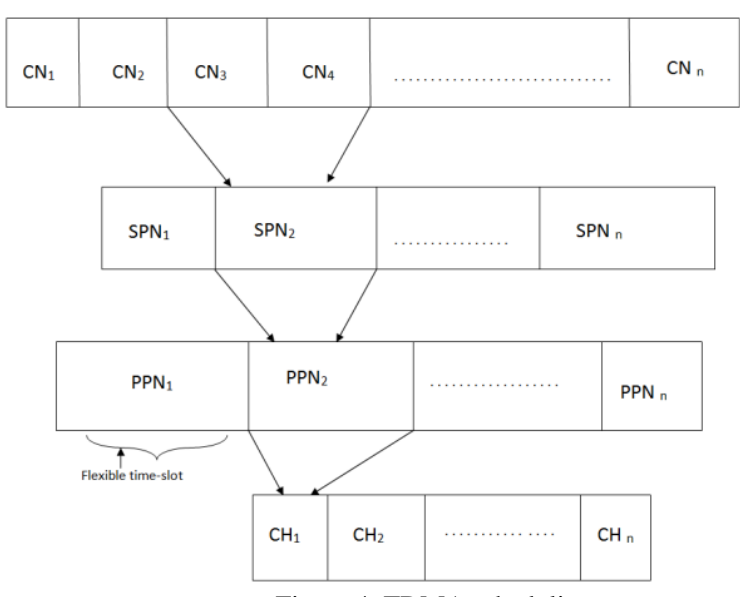

Figure 4. TDMA scheduling

In dynamic TDMA if the particular child-node's energy is reduced the neighboring node which has greater energy level get hold of the data and send in its time-slot i.e TDMA is flexible[23-24].

Frame consists of time slots and each slot can deliver $k$ packets. If buffer has q-packet the time-slot may vary with that of time slot, The number of packets may be:

1. Equal to time-slot(q=time-slot $)$

2. Less than the time-slot $(\mathrm{q}<$ time-slot $)$

3. Greater than the time-slot $(q>$ time-slot $)$

The time-slot can be increased or decreased accordingly.

The changes in the time slot can be done using the harmonic equation

$$
\mathrm{H}=\frac{U}{\sum_{i=0}^{n} \frac{1}{x_{i}}}
$$

Where $\mathrm{H}$ is harmonic mean, $\mathrm{n}$ is the total number of sensor nodes, $\mathrm{x}_{\mathrm{i}}$ is amount of packets that did not send in particular time-slot. The rules to be followed are:

1. If $\mathrm{H}>1$, then the time slot must be increased. Higher value of $\mathrm{H}$ greater the stability

2. If $\mathrm{H}<1$, then the time-slot should be decreased.

3. If none of the time-slot are free,i.e if the packets are equal to the time-slot then no changes are required.

4. If $\mathrm{H} \leq 0$ there is no variation in time-slot.

\section{FAULT TOLERANT :}

Fault tolerance algorithm solves the energy depletion problem of the Cluster Head node by attaining immediate remedy for energy outage nodes[25-27].

Whenever the Cluster Head nodes suffer from the energy depletion it will be replaced with another $\mathrm{CH}$ node. Every node has a chance to become Cluster Head at once, the probability to become a Cluster Head is $1 / \mathrm{CH}$. However the new node which is substitute to the Cluster Head do not effect the overall cluster and execute within the specified time-slot.
Cluster Head with maximum energy level is selected as new Cluster Head. The resignation message is sent by the old Cluster Head to its members and it continues as normal node . The message contains the ID of the old Cluster Head and new Cluster Head, the members of the cluster join the new Cluster Head and update their information to it.Further the new active Cluster Head starts functioning.

I. Fault tolerant Sensor Node:

In fault tolerant sensor node model, redundant nodes are assumed to be in standby mode. The redundant node become active only when the active node are detected with fault. For redundant node to be active parameters like Coverage factor, sensor failure rate and link failure rate are considered for Markov model

i. Coverage factor:The probability that active node is detected correctly if found faulty and replaced is defined by the Coverage factor $\mathrm{c}$.

$$
\mathrm{c}=\mathrm{c}_{\mathrm{e}}-\mathrm{c}_{\mathrm{u}}
$$

where $c_{e}$ is the accuracy in detection the fault sensors and $c_{u}$ defines the probability failure for replacement of the identified defective sensor nodes. The exactness depends upon the average number of sensor neighbor nodes and the probability of sensor node failure $f$. Empirical relation for $\mathrm{c}_{\mathrm{e}}$ : $c_{e} \leq 1$ is given by:

$$
\mathrm{c}_{\mathrm{e}}=\frac{e \mathrm{x}(1-f)}{e^{\left(e /(M(f))^{1 / M(f)}\right.}+(1-e / M(f))^{e}}
$$

where $\mathrm{M}(f)$ is function of $f$ equation that determines the fault detection accuracy.

ii. Sensor Failure rate: It can be characterized by the exponential distribution with a failure rate $\lambda_{\mathrm{ft}}$ over $\mathrm{t}_{\mathrm{s}}$ period. Exponential distribution portion in the all-around approximation specifies failure i.e constant failure rate over a small time.

The exponential failure rate is given by:

$$
\operatorname{Fs}\left(\mathrm{t}_{\mathrm{s}} ; \lambda_{\mathrm{ft}}\right)=f=1-\exp \left(-\lambda_{\mathrm{ft}} \mathrm{t}_{\mathrm{s}}\right)
$$

Model:

The fault sensor node consists 2 states:state1(good/strong)and state $0(\mathrm{bad} /$ weak $)$. The differential equation that describe the Markov model is given by:

$$
\begin{aligned}
& P_{1}^{\prime}(t)=-\lambda_{\mathrm{a}} \mathrm{P}_{1}(\mathrm{t}) \\
& P_{0}^{\prime}(t)=-\lambda_{\mathrm{a}} \mathrm{P}_{0}(\mathrm{t})
\end{aligned}
$$

Where $\mathrm{P}_{\mathrm{i}}(\mathrm{t})$ denotes the probability of the sensor node and $P_{i}^{\prime}(t)$ denote the first order probability. $\lambda_{\mathrm{a}}$ denotes the failure rate of active sensor. 
By applying initial conditions $\mathrm{P}_{1}(0)=0$ and $\mathrm{P}_{0}(0)=0$ to equation we get

$$
\begin{aligned}
& \mathrm{P}_{1}(\mathrm{t})=e^{-\lambda_{a} t} \\
& \mathrm{P}_{0}(\mathrm{t})=1-e^{-\lambda_{a} t}
\end{aligned}
$$

The reliability of sensor node is given by:

$\mathrm{L}_{\mathrm{s}(\mathrm{t})}=1-\mathrm{P}_{0}(\mathrm{t})=\mathrm{P}_{1}(\mathrm{t})==e^{-\lambda_{a} t}$

The MTTF of sensor node is given by:

$\operatorname{MTTF}_{\mathrm{s}}=\int_{0}^{\infty} \mathrm{L}_{\mathrm{s}(\mathrm{t})}=1 / \lambda_{a}$

Where MTTF is mean time to failure

The average failure rate is given by:

$$
\lambda_{\mathrm{s}}=\frac{1}{\mathrm{MTTFs}}=\lambda_{a}
$$

In 2 state node model the inactive sensor node becomes active only after node has been detected as fault by the fault detection algorithm. The Markov model as shown below figure represent the number of sensors which are good, for Markov model the differential equation is given by:

$$
\begin{aligned}
& P_{2}^{\prime}(t)=-\lambda_{a} P_{2}(t) \\
& P_{1}^{\prime}(t)=-\lambda_{a} c P_{2}(t)-\lambda_{a} P_{1}(t) \\
& P_{0}^{\prime}(t)=-\lambda_{a}(1-c) P_{2}(t)+\lambda_{a} P_{1}(t)
\end{aligned}
$$

State 1 and state 2 indicate the active state and state 0 indicate the failed state. Where (1-c) is the probability of fault nodes that cannot be recovered. Therefore by solving the above equation by putting the initial condition conditions $\mathrm{P}_{2}(0)=0$ $\mathrm{P}_{1}(0)=0$ and $\mathrm{P}_{0}(0)=0$ we get:

$$
\begin{aligned}
& \mathrm{P}_{2}(\mathrm{t})=e^{-\lambda_{a} t} \\
& \mathrm{P}_{1}(\mathrm{t})=\mathrm{c} \lambda_{\mathrm{a}} \mathrm{t} e^{-\lambda_{a}^{t}} \\
& \mathrm{P}_{0}(\mathrm{t})=1+\mathrm{P}_{1}(\mathrm{t})+\mathrm{P}_{2}(\mathrm{t})
\end{aligned}
$$

Fault tolerant node reliability is given by:

$$
\mathrm{L}_{\mathrm{st}(\mathrm{t})}=1-\mathrm{P}_{0}(\mathrm{t})=\mathrm{P}_{1}(\mathrm{t})+\mathrm{P}_{2}(\mathrm{t})=e^{-\lambda_{a} t}+\mathrm{c} \lambda_{\mathrm{a}} \mathrm{t} e^{-\lambda_{a}^{t}}
$$

The MTTF of sensor node is given by:

$$
\mathrm{MTTF}_{\mathrm{st}}=\int_{0}^{\infty} \mathrm{L}_{\mathrm{st}(\mathrm{t})}=1 / \lambda_{a}+\mathrm{c} / \lambda_{a}
$$

The average failure node is given by:

$$
\lambda_{\mathrm{st}}=\frac{1}{M T T F_{s t}}
$$

where $\lambda_{\text {st }}$ denote the average failure rate.

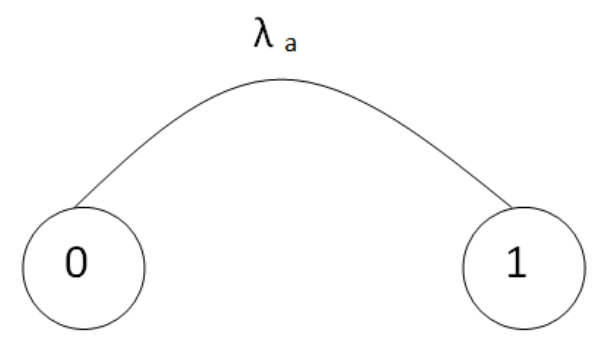

Figure 5 : A 2 state non-fault sensor Markov model

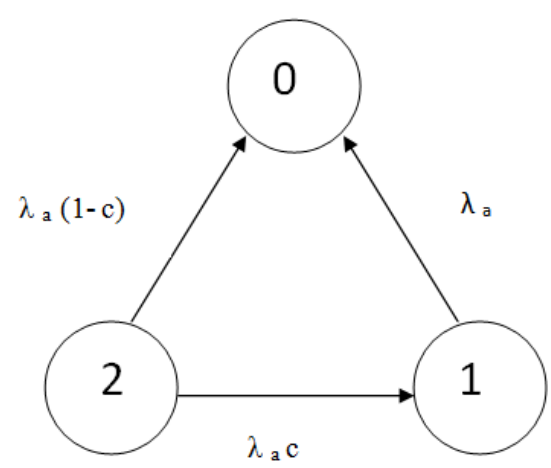

Figure 6.A Fault tolerant sensor Markov model

For sensor fault tolerant model the average failure node mainly depends upon the $f$ because the accuracy depends upon the fault detection algorithm. Hence the fault nodes are calculated for various values using the Markov model.

\section{iii. LINK FAILURE:}

Link failure adversely affects the network performance and reliability.Link failure(path loss) occurs between the sensor nodes and the sink nodes, hence longest path should be selected for transmission and this causes energy consumption and therefore longest transmission delay.

In link failure model by taking into consideration of energy equations fault nodes are given by:

$$
\mathrm{P}_{\mathrm{L}}=\left(1-e^{-\left(Y+Z \cdot d^{2}\right) t}\right)\left(1-e^{-\lambda(f t) t}\right)
$$

$$
\text { Where } \mathrm{Y}=\frac{2 n_{o} E_{\text {elec }}}{E_{\text {ini }}}, \mathrm{Z}=\frac{n_{o} \varepsilon_{\text {amp.k}}}{E_{\text {ini }}}
$$

$\mathrm{Y}$ and $\mathrm{Z}$ are link between the two nodes

In clustering scheme, if cluster meets $t \geq t_{\text {min }}$ all the nodes satisfy the condition $\mathrm{c}_{\min } \leq \mathrm{c} \leq \mathrm{c}_{\max }$ and if the distance between the adjacent nodes satisfy the distance condition then they are meant to meet improvement of link failure $d_{\min } \leq d \leq d_{\max }$.

Proof: 
According to condition $\mathrm{t} \geq \mathrm{t}_{\min }$

$\mathrm{P} \geq\left(1-e^{-\left(Y+Z \cdot d_{\text {max }}^{2}\right) t_{\min }}\right)\left(1-e^{\frac{\lambda_{f t}}{1+c_{\min }} t \min }\right)$

Since $c_{\min } \leq \mathrm{c} \leq \mathrm{c}_{\max }$ and $\mathrm{d}_{\min \leq} \mathrm{d} \leq \mathrm{d}_{\max }$ we get

$=\left(1-e^{\frac{\lambda_{f t}}{1+c_{\max }} t}\right) \leq\left(1-e^{\frac{\lambda_{f t}}{1+c} t}\right) \leq\left(1-e^{\frac{\lambda_{f t}}{1+c_{\max }} t}\right)$

$=\left(1-e^{-\left(Y+Z \cdot d_{\min }^{2}\right) t}\right) \leq\left(1-e^{-\left(Y+Z \cdot d^{2}\right) t}\right) \leq\left(1-e^{-\left(Y+Z \cdot d_{\max }^{2}\right) t}\right)$

After reducing we get

$\left(1-e^{-\left(Y+Z \cdot d_{\min }^{2}\right) t}\right)\left(1-e^{\frac{\lambda_{f t}}{1+c_{\max }} t}\right) \leq \mathrm{P} \leq$

$\left(1-e^{-\left(Y+Z . d_{\max }^{2}\right) t}\right)\left(1 e^{\frac{\lambda_{f t}}{1+c_{\max }} t}\right)$

Thus we obtain

$\mathrm{P} \geq\left(1-e^{-\left(Y+Z \cdot d_{\text {max }}^{2}\right) t_{\min }}\right)\left(1-e^{\frac{\lambda_{f t}}{1+c_{\min }} t \min }\right)$

Therefore the above model meets required lifetime and link fault-tolerance.

\section{FAULT-TOLERANT MODEL USING FUZZY LOGIC:}

To recover sensor and link failure distributed sensor network using Fuzzy logic is proposed [28-30] .Fuzzy inference is used to procure conclusion from environment known or assumed true values. Using Euclidian distance we calculate distance between two nodes

$$
\mathrm{d}=\sqrt{(x i-x j)^{2}+(y i-y j)^{2}}
$$

Path loss can be determined by considering following parameters. Let $C_{i}$ be the discrete time capacity and, $B$ is the bit-rate $(\mathrm{Hz})$ of channel and the signal to noise ratio as SNR. Capacity of channel can be determined as:

$\mathrm{C}_{\mathrm{i}}=\mathrm{B} \log _{2}(1+\mathrm{SNR})$

Link efficiency is defined as:

$\mathrm{L}_{\mathrm{EFF}}=\mathrm{C}_{\mathrm{i}} / \mathrm{E}$

Where $\mathrm{E}$ is energy of node.

Functions:

1. Fuzzylogic $\left(\mathrm{L}_{\mathrm{EFF}}, \mathrm{s}_{\mathrm{i}}\right)$

2. $\operatorname{Send}\left(\right.$ data, $\left.\mathrm{s}_{\mathrm{i}}\right)$

3. $\operatorname{Receive}\left(\mathrm{s}_{\mathrm{i}}, \mathrm{s}_{\mathrm{n}}\right)$
1. Consider randomly deployed sensors

2. If $\left(\mathrm{E}_{\mathrm{T}} \geq \mathrm{T}_{\mathrm{E}}\right.$ and $\left.\mathrm{L}_{\mathrm{EFF}} \geq \mathrm{T}_{\mathrm{EFF}}\right)$;
a. Node is Active;
b. else
c. Node is Dead;
d. end if
e. end for

3. Data transmission and processing of nodes in DSN is as follows:
a. $\operatorname{If}\left(\left(\mathrm{s}_{\mathrm{i}}\right)=\right.$ TRUE $)$
b. Data=Fuzzylogic $\left(\mathrm{L}_{\mathrm{EFF}}, \mathrm{s}_{\mathrm{i}}\right)$
c. else
d. Select alternate path for better link efficiency
e. end if

Selection of efficient fault detection in the DSNs number.

$\mathrm{T}_{\mathrm{E}}$ is threshold energy and $\mathrm{T}_{\mathrm{EFF}}$ is threshold level of link efficiency.

\section{SIMULATION}

Comparsion of fault-tolerance model is generated using Poisson distribution.BA model,Model 1,Model2 and Distributed fuzzy logic model are compared to check the fault-tolerance and incursion tolerance.Initally all the network models are same and during the fault tolerance ,failure nodes are removed at every round.

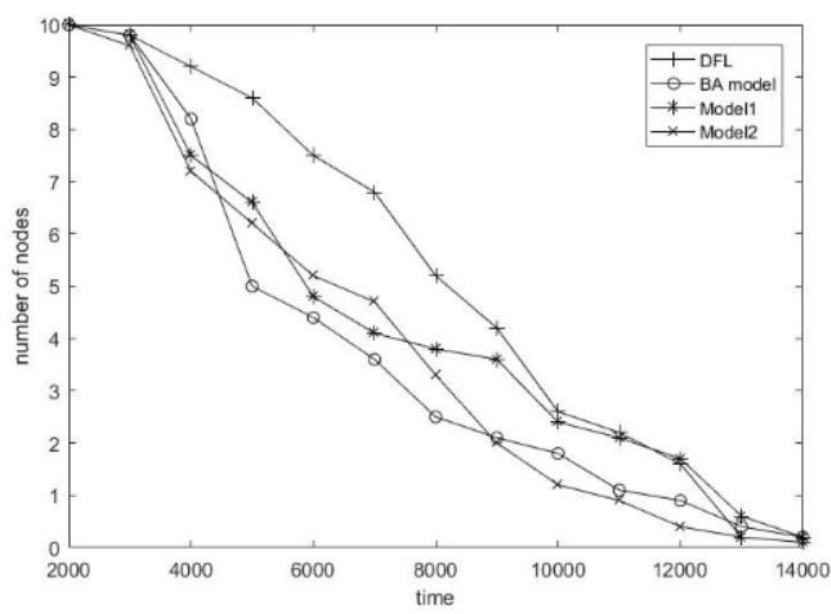

Figure 7:Fault-Tolerance Comparison

From Figure 7 we can observe that BA model is slightly greater than other model at around 3900 round.As time runs the performance of BA model is completely down and our Proposed model DFL has better results compared to other 3 Models. This shows that DFL model is superior then other model for detection and replacement of the nodes.

Algorithm for Distributed Fuzzy Logic 


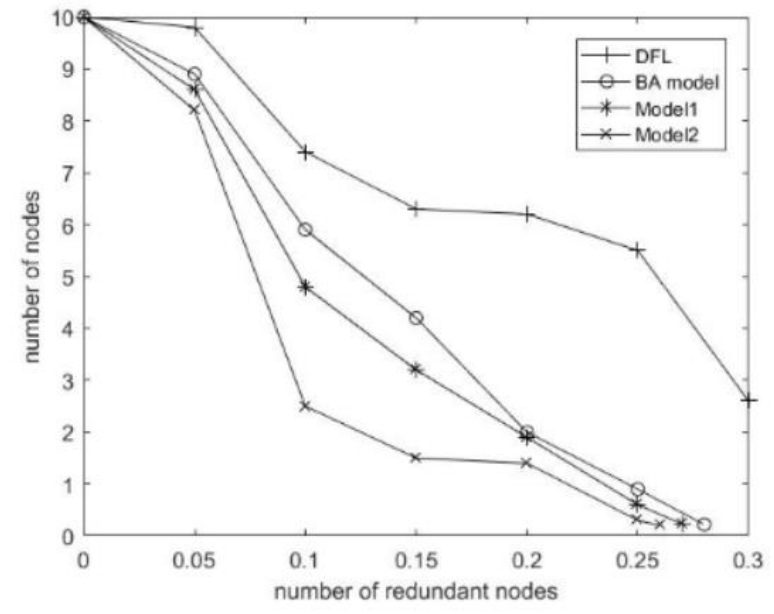

Figure 8:Incursion tolerance Comparison

In Incursion tolerance model the nodes $\mathrm{CH}$ are randomly taken out from range 0 to 0.3 .From Figure 8 it can be seen that DFL model is stronger and cautious against attack. The network lifetime in comparison with other model DFL has good and balanced.

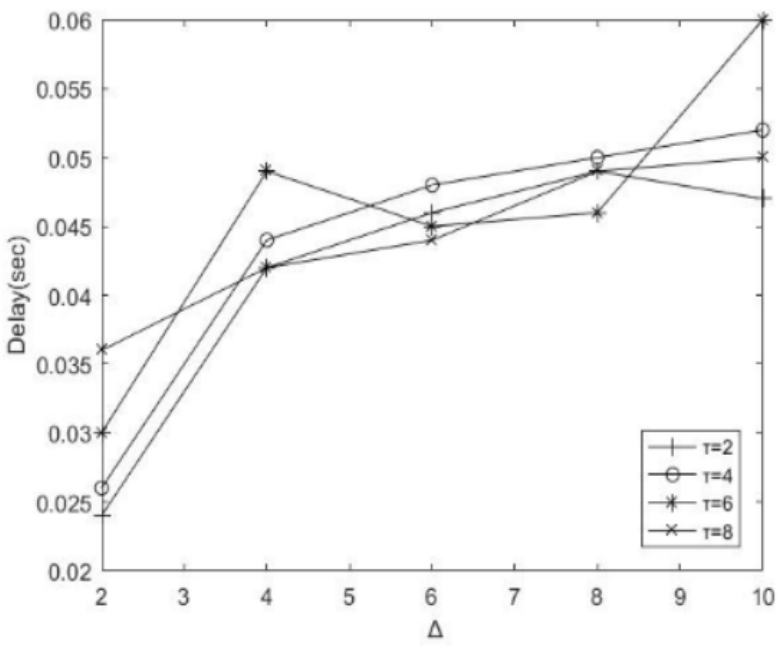

Figure 9:End to End delay

Speed ,Power and Bandwidth are necessary parameters in order to forward the small packets of information. For various values of $\zeta, \Delta$ graph is shown.

$\zeta$ is considered as low speed $\Delta$ is considered as high speed.

Therefore for various values of $\zeta$ the end to end delay is calculated to determine the speed and power required to send the packets of data at the receiver side.

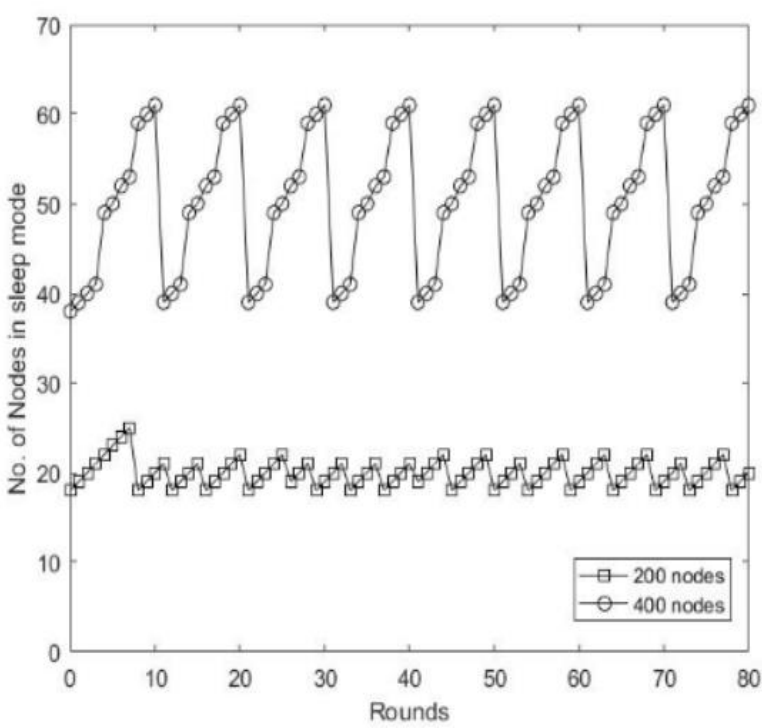

Figure 10: Number of sleep nodes in Network

In Figure 10 we can observe that number of sleep nodes in high density is larger than number of sleep nodes in low density. This shows that number of nodes in sleep mode are high .The number of sleep nodes is stable in higher density which indicates there is stability in energy consumption.

\section{CONCLUSION:}

This paper presents fuzzy logic technique which is used for selection of redundant nodes after formation of parent-child relation. The TDMA technique used here is dynamic TDMA which has flexible time slots, this make advantages to nodes which have large amount of data.

Also by using Fuzzy logic we can detect the fault nodes and replace them early using distributed fuzzy technique.The above graphs show the better results using DFL.

\section{REFERENCES:}

[1] Dilip Kumar, Trilok C. Aseri , R.B. Patel, EEHC: Energy efficient heterogeneous clustered scheme for wireless sensor networks Computer Communications 32 (2009).

[2] Wendi B. Heinzelman, Anantha P.Chandrakasan, Hari Balakrishnan, An Application-Specific Protocol Architecture for Wireless Microsensor Networks, ieee transactions on wireless communications, vol. 1, no. 4, october 2002 .

[3] Ozlem Karaca · Radosveta Sokullu · Neeli R. Prasad · Ramjee Prasad ,Application Oriented Multi Criteria Optimization in WSNs Using on AHP,Wireless Pers Commun (2012) 65.

[4] Yan Wu, Sonia Fahmy, Ness B. Shroff, Optimal Sleep/Wake Scheduling for Time-Synchronized Sensor Networks with QoS Guarantees, (C2006 ieee.

[5] Runze Wan, Jianjun Lei, Qingwei Xu, Ximei Gou, An Energyefficient Unequal Clustering Algorithm in Wireless SensorNetworks ,Advanced Materials Research Vol. 629 (2013).

[6] Nguyen Duy Tan, Nguyen Dinh Viet ,SSTBC: Sleep Scheduled and Tree-Based Clustering Routing Protocol for Energy-Efficient in Wireless Sensor Networks, The 2015 IEEE RIVF International Conference on Computing \& Communication Technologies.

[7] Rick W. Ha a, Pin-Han Ho , X. Sherman Shen, Junshan Zhang, Sleep scheduling for wireless sensor networks via network flow model Computer Communications 29 (2006).

[8] Avinash More a, Vijay Raisinghani, A node failure and batteryaware coverage protocol for wireless sensor networks, Computers and Electrical Engineering (2017). 
[9] Bahia Zebbane, Manel Chenait and Nadjib Badache, Exploiting Node Redundancy for Maximizing Wireless Sensor Network Lifetime, (C2013 IEEE.

[10] In`es El Korbi, Yacine Ghamri-Doudaney, Rimel Jazi and Leila Azouz Saidane ,Coverage-Connectivity based Fault Tolerance Procedure in Wireless Sensor Networks @2013 IEEE.

[11] Nafaâ Jabeura, Ahmed Nait Sidi Mohb, Mohamed Mahdi Barkiaa A Bully Approach for Competitive Redundancy in Heterogeneous Wireless Sensor Network, Procedia, Computer Science 83 ( 2016 ).

[12] arslan munir, joseph antoon, ann gordon-ross, modeling and analysis of fault detection and fault tolerance in wireless sensor networks acm transactions on embedded computing systems, vol.14, no. 1, article 3, publication date: january 2015.

[13] Mohamed Younis, Sookyoung Lee, Izzet Fatih Senturk and Kemal Akkaya, Topology Management Techniques for Tolerating Node Failure, (C) Springer-Verlag Berlin Heidelberg 2014.

[14] Shu-Yin Chiang and Jing-Long Wang, Routing Analysis Using Fuzzy Logic Systems in Wireless Sensor Networks, (C) SpringerVerlag Berlin Heidelberg 2008.

[15] Beatrice Lazzerini+, Francesco Marcelloni+, Massimo Vecchio, Silvio Croce+, Emmanuele Monaldi, A Fuzzy Approach to Data Aggregation to Reduce Power Consumption in Wireless Sensor Networks, (C2006 IEEE.

[16] Haixia Peng_y, Shuaizong Si_, Xuemin (Sherman) Sheny, Hai Zhao, Energy-Efficient and Scalable Clustering Scheme for Wireless Sensor Networks, (2015 IEEE.

[17] Lifang Liu,1 Xiaogang Qi,2,3 Jilong Xue,4 andMande Xie A Topology Construct and Control Model with Small-World and Scale-Free Concepts for Heterogeneous Sensor Networks, Hindawi Publishing CorporationInternational Journal of Distributed Sensor Networks Volume 2014.

[18] HaoranLiua,RongrongYina,,BinLiua,YaqianLi, Ascalefreetopologymodelwithfault-toleranceandintrusiontoleranceinwirelesssensornetworks, Computers and Electrical Engineering 000 (2016).

[19] K.S.Arikumar Dr.V.Natarajan L.Sushma Clarence, M. Priyanka, Efficient Fuzzy Logic Based Data Fusion in Wireless Sensor Networks, 2016 Online International Conference on Green Engineering and Technologies (IC-GET).

[20] Ashfaq Ahmad1, Nadeem Javaid1, Muhammad Imran2, Mohsen Guizani3, Ahmad A. Alhamed, An advanced energy consumption model for terrestrial wireless sensor networks, (C2016 IEEE.

[21] Jong-Myoung Kim, Seon-Ho Park, Young-Ju Han and TaiMyoung Chung, CHEF: Cluster Head Election mechanism using Fuzzy logic in Wireless Sensor Networks, Feb. 17-20, 2008 ICACT 2008

[22] Indranil Gupta, Denis Riordan, Srinivas Sampalli, Cluster-head Election using Fuzzy Logic for Wireless Sensor Networks, (C) 2005 IEEE.

[23] Winnie Louis Lee, Amitava Datta, and Rachel Cardell-Oliver, FlexiMAC: A flexible TDMA-based MAC protocol for faulttolerant and energy-efficient wireless sensor networks, 2006 IEEE.

[24] Sungrae Cho, Kalyani Kanuri, Jin-Woong Cho, Jang-Yeon Lee, and Sun-Do June, Dynamic Energy Efficient TDMA-based MAC Protocol forWireless Sensor Networks, (C) 2005 IEEE.

[25] Vinod Kumar Menaria, S.C. Jain, A. Nagaraju, A fault tolerance based route optimisation and data aggregation using artificial intelligence to enhance performance in wireless sensor networks, Int. J. Wireless and Mobile Computing, Vol. 14, No. 2, 2018.

[26] C.Sivaraj, P.J.A.Alphonse, T.N.Janakiraman, An Energy Efficient Fault tolerance Nested Clustering Algorithm for Routing in Wireless SensorNetworks,IEEE.

[27] Mohamed El Fissaoui - Abderrahim BeniHssane Mostafa Saadi2, Energy efficient and fault tolerant distributed algorithm for data aggregation in wireless sensor networks, (C) SpringerVerlag GmbH Germany, part of Springer Nature 2018.

[28] Sara Behzadi1, Mohammad Azad, Fault-tolerant in wireless sensor networks using fuzzy logic, International Research Journal of Applied and Basic Sciences (C) 2014.

[29] Shahab Tohidy, Ali Khaki Sedigh, Fault Tolerant Fuzzy Control Allocation for Overactuated Systems, 2013 13th Iranian Conference on Fuzzy Systems (IFSC).
[30] Shaocheng tong,Yue Wang, Fault tolerance Control method based on Fuzzy logic system,2008 IEEE. 